\title{
Double mutant fibrillin-1 (FBN1) allele in a patient with neonatal Marfan syndrome
}

\author{
Mei Wang, Priya Kishnani, Martha Decker-Phillips, Stephen G Kahler, Yuan-Tsong Chen, \\ Maurice Godfrey
}

\begin{abstract}
It is now well established that defects in fibrillin-1 (FBN1) cause the variable and pleiotropic features of Marfan syndrome (MFS) and, at the most severe end of its clinical spectrum, neonatal Marfan syndrome (nMFS). Patients with nMFS have mitral and tricuspid valve involvement and aortic root dilatation, and die of congestive heart failure, often in the first year of life. Although mutations in classical MFS have been observed along the entire length of the FBN1 mRNA, mutations in nMFS appear to cluster in a relatively small region of FBN1, approximately between exons 24 and 34 . Here we describe the appearance of two FBN1 mutations in a single allele of an infant with nMFS. The changes were within six bases of each other in exon 26. One was a T3212G transversion resulting in an $I 1071 S$ amino acid substitution and the second was an A3219T transversion resulting in an E1073D amino acid substitution. This is the first reported double mutant allele in FBN1. (FMed Genet 1996;33:760-763)
\end{abstract}

Key words: Marfan syndrome; fibrillin; mutation.

Recently, in describing the identification of two cystic fibrosis transmembrane conductance regulator (CFTR) mutations in a single allele, Savov et $a l^{1}$ asked whether double mutant alleles were rare. Analysis of the CFTR gene in several populations has shown the appearance of double mutant alleles. ${ }^{1-4}$ In fact, different clinical phenotypes have been associated with the presence of double mutant alleles. Similar double mutant alleles have been observed in the acid $\beta$-glucosidase gene in Gaucher disease $e^{5}$ and in the p53 gene in some human tumours. ${ }^{6}$

Marfan syndrome (MFS) is an autosomal dominant heritable connective tissue disorder manifested by abnormalities in the skeletal, ocular, and cardiovascular systems. ${ }^{7}$ Neonatal Marfan syndrome (nMFS) is at the most severe end of the phenotypic expression of MFS. ${ }^{8-13}$ Patients with nMFS share many of the skeletal features seen in classical MFS, but their cardiovascular phenotype includes acute multivalvular disease that is rarely seen in classical MFS. These patients often die of congestive heart failure within the first year of life.

It is now well known that defects in the gene encoding fibrillin-1 (FBN1) cause a wide spec- trum of microfibrilopathies. These include MFS, ${ }^{14-2223-30}$ nMFS, ${ }^{23-25}{ }^{30}{ }^{31}$ a family with relatively mild skeletal findings, ${ }^{32}$ ectopia lentis, ${ }^{24}{ }^{33}$ and late onset aortic aneurysms. ${ }^{34}$ Interestingly, all documented mutations in nMFS patients have clustered between exons 24 and 34 . Here we describe a double mutant allele in a nMFS patient. The mutations, in exon 26, are a $\mathrm{T} 3212 \mathrm{G}$ transversion that results in an I1071S amino acid substitution and an A3219T transversion resulting in an E1073D amino acid substitution. Although probably caused by a single mutational event, this is the first reported double mutant fibrillin-1 allele.

\section{Materials and methods}

CASE REPORT

A black female infant with clinical features of severe $\mathrm{nMFS}$ was born to a 28 year old primigravida at 34 weeks' gestation. Cranial malformation, diaphragmatic abnormality, limb anomaly, and tricuspid and pulmonary insufficiency were noted prenatally. Birth weight, length, and head circumference were normal. She had scaphocephaly, plagiocephaly, and widely open anterior and posterior fontanelles. The ears were large with flat pinnae. The chest showed pectus carinatum and profound left thoracolumbar scoliosis. Cardiac findings were significant for dextrocardia. There were bilateral flexion contractures of the elbows, wrists, hips, knees, and ankles. She had arachnodactyly, camptodactyly, loose redundant skin, and decreased muscle mass. Ophthalmological evaluation showed bilateral optic nerve colobomata with no lens subluxation. Echocardiogram, at birth, showed situs solitus, dextrocardia, and prolapsing tricuspid and mitral valves, with tricuspid and mitral insufficiency and right ventricular hypertrophy. She had eventration of the right hemidiaphragm which was surgically corrected at $1 \frac{1 / 2}{2}$ months. Chromosome analysis from amniocytes, blood lymphoblasts, and skin fibroblasts was $46, \mathrm{XX}$. The patient's hospital course was complicated by several episodes of spontaneous pneumothorax requiring persistent intubation. She died at the age of 3 months following a tracheostomy procedure. Permission for necropsy was not granted.

\section{DNA EXTRACTION}

Extraction of DNA from dermal fibroblasts was carried out as previously described. ${ }^{25}$ 
POLYMERASE CHAIN REACTION

Amplification of genomic DNA from the patient and controls was performed using intron primers flanking exon 26: forward 5'AAT TAA GGC TGT CCT GAG AC 3' and reverse 5' CAT GGA ATC CTT CTC TTT CTG 3 ' at the following conditions: denaturing $94^{\circ} \mathrm{C} / 1.5$ minutes, annealing $58^{\circ} \mathrm{C} / 2.5 \mathrm{~min}-$ utes, and extension $72^{\circ} \mathrm{C} / 2.5$ minutes for 30 cycles.

\section{HETERODUPLEX ANALYSES}

Amplified products were screened for the presence of possible mutations using Mutation Detection Enhancement (MDE) (FMC Corp) gels. Primer sequences and amplification conditions were as described by Nijbroek et al. ${ }^{30}$ In order to maximise the formation of heteroduplexes, $10 \mu \mathrm{l}$ of PCR product was mixed with 2 $\mu \mathrm{l}$ of gel loading buffer ( $50 \%$ sucrose, $0.6 \%$ xylene cyanol, $0.6 \%$ bromophenol blue) and incubated at $95^{\circ} \mathrm{C} /$ three minutes, $75^{\circ} \mathrm{C} /$ five minutes, $55^{\circ} \mathrm{C} /$ five minutes, and $37^{\circ} \mathrm{C}$ for at least five minutes until all samples were loaded on the gel. Samples were resolved on $0.5 \times$ $\mathrm{MDE}$ gels at 800 volts for approximately five hours according to the manufacturer's protocol. After electrophoresis, gels were stained in a solution of $0.6 \times \mathrm{TBE}$ containing $1 \mu \mathrm{g} / \mathrm{ml}$ ethidium bromide and DNA was visualised under UV light.

A

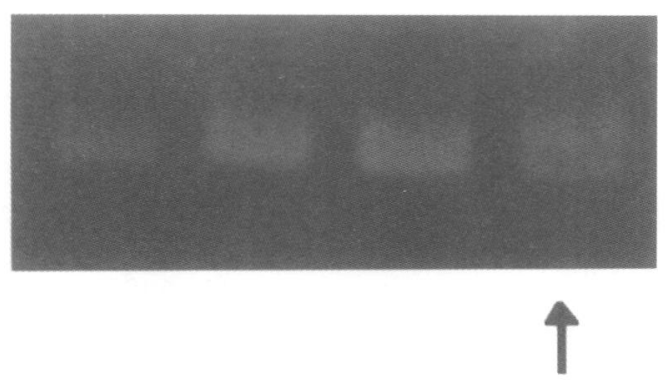

B
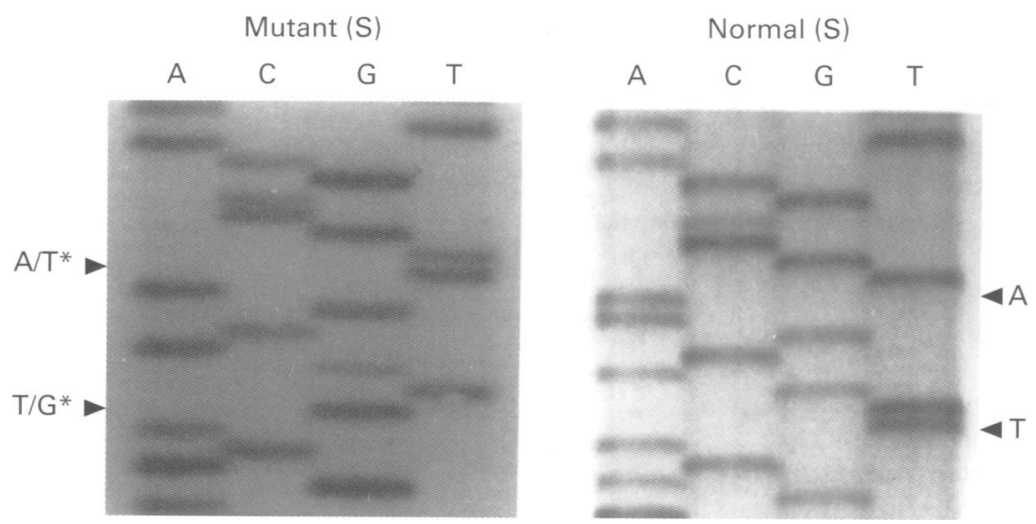

Figure 1 (A) Mutation detection enhancement (MDE) gel analysis of an amplicon of exon 26 from genomic DNA. The heteroduplex band can be seen in the lane from the patient (arrow). The remaining lanes represent analysis of the same amplified region in controls, but do not exhibit heteroduplex formation. (B) Sequence analysis of cloned fragments showed two transversions within seven nucleotides, T3212G and A3219T, in the "mutant" clone when compared to the "normal" clone.
CLONING AND SEQUENCING

Cloning and sequencing was performed as previously described. ${ }^{25}$

\section{Results}

Genomic DNA, amplified with intron primers to exon 26, showed heteroduplex formation when run on an MDE gel (fig 1A). Direct sequencing of the amplified fragment showed two nucleotide substitutions (data not shown). In order to determine whether both changes were on a single allele, the amplicon was cloned and subsequently sequenced. Sequence analysis of the clones showed that both the T3212G and A3219T transversions were on the same FBN1 allele (fig 1B). The former mutation resulted in an I1071S amino acid substitution and the latter transversion resulted in an E1073D amino acid substitution. Study of the parents confirmed that neither harboured any of the mutations (data not shown). Studies using several highly informative markers were also consistent with paternity.

\section{Discussion}

It is becoming increasingly evident that a clustering of some FBN1 mutations in what Kainulainen et $a l^{4}$ have called "the neonatal region" results in disease at the most severe end of the Marfan syndrome phenotype spectrum, namely nMFS. Recent studies have expanded the region to include approximately exons 24 to 34 of FBN1. ${ }^{23-25} 3031$ Importantly, not all patients with mutations in the "neonatal region" have nMFS. Some have classical MFS, but at the severe end of the spectrum, ${ }^{14}{ }^{35}$ while others have missense mutations that cause typical or mild MFS. ${ }^{30}{ }^{31}$ A compound heterozygous MFS patient, whose parents had classical MFS, has already been described. ${ }^{36} \mathrm{His}$ two mutations caused very severe disease and early death.

The patient reported here had the typical cardiovascular features seen in nMFS, but also had dextrocardia. There are three major causes of dextrocardia: (1) situs inversus, a mirror image inversion of all the normally asymmetrical structures; (2) dextroversion, failure of the cardiac apex to rotate from right to left; and (3) dextroposition, a mechanical shift of the heart to the right owing to either a mass effect in the left chest or lack of normal lung volume in the right chest. ${ }^{37}$ Echocardiograms and plain chest and abdominal radiographs showed the position of the liver, spleen, stomach, and heart in our patient. The cardiac situs was determined to be solitus (atria were in the correct right-left orientation). Also, there was no abdominal heterotaxia. This finding rules out laterality disorders, such as Kartagener syndrome and the asplenia and polysplenia disorders. We believe this patient had dextroposition secondary to her severely misshapen thoracic cage, despite the raised right hemidiaphragm. Unfortunately, no necropsy was performed that would have confirmed this suspicion.

Although many hundreds of CFTR mutations have been reported, ${ }^{38}$ from the thousands of CF patients screened, few double mutant alleles have been identified. Savov et $a l^{1}$ 
correctly point out that once the common mutations have been identified in patients screening stops. It is indeed possible that if the entire CFTR was screened in all CF patients, additional double mutant alleles would be uncovered. Since almost all MFS patients and families have unique FBN1 mutations, most protocols call for the screening of the entire coding region and flanking splice junctions. The recent publication of intron primer pairs for all 65 FBN1 exons has greatly aided the effort to identify mutations in MFS patients and in those with overlapping phenotypes. ${ }^{30}$

In our routine screening through the neonatal region first and subsequently the entire complement of the remaining FBN1 exons, we identified a nMFS patient with two changes in FBN1. Since the initial sequencing was performed directly from PCR amplicons, we were unsure whether both changes were in the same allele. Cloning of the PCR products showed that both changes were in a single allele. It was still unclear whether one change was a polymorphism and the issue of paternity also needed to be addressed. Paternity was confirmed by examining four highly polymorphic markers. Amplification and sequencing of parental DNA showed that neither parent harboured either the T3212G or A3219T transversions. Thus, both changes had occurred sporadically and, given their proximity, were most probably a single mutational event. Conformational studies of the folding region of FBN1 some 50 bases upstream and downstream from the mutations do not show any particularly stable DNA secondary structures that might interfere with replication. It may be significant that both mutations are transversions, however. Both of the substituted positions are conserved in the precursor epidermal growth factor (EGF)-like calcium binding domains of FBN1. The I1071S amino acid substitution appears to be the most significant, since the substitution of a hydrophobic residue for a polar amino acid may disrupt the hydrophobic core of fibrillin. The E1073D amino acid substitution appears, on the surface, to be fairly conservative, since both aspartate and glutamate are negatively charged with relatively small side chains. However, the glutamic acid is invariant in the FBN1 EGF-like domains and is adjacent to one of the cysteine residues that are also invariant. The substitution of aspartic acid for glutamic acid may influence the formation of disulphide bridges. A substitution at the 1073 residue causing nMFS has in fact been described. ${ }^{30}$ In that case, however, the substitution for glutamic acid was for lysine, a basic amino acid with a much larger side chain. It is likely that both the I1071S and E1073D amino acid substitutions played a role in altering the structure of fibrillin-1 and the microfibrils in this case of nMFS. Double mutant alleles are probably rare in most disorders, but, when present, may influence the course of the clinical outcome.

We thank Drs L James Maher III and Simon Sherman for helpful discussions and James D Birrell and Joe Edwards for excellent photographic assistance. This work was supported in part by grants from the National Heart, Lung, and Blood Institute of the National Institutes of Health HL48126 (MG) and a Clinical Research Grant 6-FY94-0012 from the March of Dimes Birth Defects Foundation (MG). MG is an Established Investigator of the American Heart Association (funding was contributed in part by the AHA Florida Affiliate)

1 Savov A, Angelicheva D, Balassopoulou A, et al. Double mutant alleles: are they rare? Hum Mol Genet 1995;4:116971 .

2 Kalin N, Dork T, Tummler B. A cystic fibrosis allele encoding missense mutations in both nucleotide binding folds of the cystic fibrosis transmembrane conductance regulator. Hum Mutat 1992;1:204-10.

3 Dork T, Wulbrand U, Richter T, et al. Cystic fibrosis with three mutations in the cystic fibrosis transmembrane conductance regulator gene. Hum Genet 1991;87:441-6.

4 Kiesewetter S, Macek M Jr, Davis C, et al. A mutation in CFTR produces different phenotypes depending on chromosomal background. Nature Genet 1993;5:274-8.

5 Latham T, Grabowski GA, Theophilus BD, Smith FI. Complex alleles of the acid beta-glucosidase gene in GauComplex alleles of the acid beta-glucosidase ge
cher disease. Am $₹$ Hum Genet 1990;47:79-86.

6 Hachiya M, Chumakov A, Miller CW, et al. Mutant p53 proteins behave in a dominant, negative fashion in vivo. Anticancer Res 1994;14:1853-9.

7 Godfrey $M$. The Marfan syndrome. In: Beighton P, ed. McKusick's heritable disorders of connective tissue. St Louis: C V Mosby, 1993:51-135.

8 Geva T, Sanders SP, Diogenes MS, Rockenmacher S, Van Praagh R. Two-dimensional and Doppler echocardioPraphic and pathologic characteristics of the infantile graphic and pathologic characteristics of the
Marfan syndrome. Am $₹$ Cardiol 1990;65:1230-7.

9 Morse RP, Rockenmacher S, Pyeritz RE, et al. Diagnosis and management of infantile Marfan syndrome. Pediatrics 1990;86:888-95.

10 Shankar KR, Hultgren MK, Lauer RM. Lethal tricuspid and mitral regurgitation in Marfan's syndrome. Am $\mathcal{f}$ Cardiol 1967;20:122-7.

11 Lababidi Z, Monzon C. Early cardiac manifestations of Marfan's syndrome in the newborn. Am Heart $\mathcal{f}$ 1981;102:943-5.

12 Phornphutkul C, Rosenthal A, Nadas AS. Cardiac manifestations of Marfan syndrome in infancy and childhood. Circulation 1973;47:587-96.

13 Sisk HE, Zahka KG, Pyeritz RE. The Marfan syndrome in early childhood: analysis of 15 patients diagnosed at less than 4 years of age. Am $\mathcal{F}$ Cardiol 1983;52:353-8.

14 Dietz HC, Cutting GR, Pyeritz RE, et al. Marfan syndrome caused by a recurrent de novo missense mutation in the fibrillin gene. Nature 1991;352:337-9.

15 Dietz HC, Pyeritz RE, Puffenberger EG, et al. Marfan phenotype variability in a family segregating a missense mutation in the epidermal growth factor-like motif of the fibrillin gene. $\mathcal{F}$ Clin Invest 1992;89:1674-80.

16 Kainulainen K, Sakai LY, Child A, et al. Two mutations in Marfan syndrome resulting in truncated fibrillin polypeptides. Proc Natl Acad Sci USA 1992;89:5917-21.

17 Godfrey M, Vandemark N, Wang M, et al. Prenatal diagnosis and a donor splice site mutation in fibrillin in a family with the Marfan syndrome. Am $\mathcal{F}$ Hum Genet 1993;53:47280.

18 Dietz HC, Saraiva JM, Pyeritz RE, Cutting GR, Francomano CA. Clustering of fibrillin (FBN1) missense mutations in Marfan syndrome patients at cysteine residues in EGF-like domains. Hum Mutat 1992;1:366-74.

19 Dietz HC, Valle D, Francomano CA, et al. The skipping of constitutive exons in vivo induced by nonsense mutations. Science 1993;259:680-3.

20 Dietz HC, McIntosh I, Sakai LY, et al. Four novel FBN1 mutations: significance for mutant transcript level and EGF-like domain calcium binding in the pathogenesis of Marfan syndrome. Genomics 1993;17:468-75.

21 Hewett DR, Lynch JR, Smith R, Sykes BC. A novel fibrillin mutation in the Marfan syndrome which could disrupt calcium binding of the epidermal growth factor-like module. Hum Mol Genet 1993;2:475-7.

22 Aoyama T, Tynan K, Dietz HC, Francke U, Furthmayr H. Missense mutations impair intracellular processing of fibrillin and microfibril assembly in Marfan syndrome. Hum Mol Genet 1993;2:2135-40.

23 Milewicz DM, Duvic M. Severe neonatal Marfan syndrome resulting from a de novo 3-bp insertion into the fibrillin gene on chromosome 15. Am F Hum Genet 1994;54:44753.

24 Kainulainen K, Karttunen L, Puhakka L, Sakai L, Peltonen L. Mutations in the fibrillin gene responsible for dominant L. Mutations in the fibrillin gene responsible for dominant Genet 1994;6:64-9.

25 Wang M, Price CE, Han J, et al. Recurrent mis-splicing of fibrillin exon 32 in two patients with neonatal Marfan syndrome. Hum Mol Genet 1995;4:607-13.

26 Hewett D, Lynch J, Child A, Firth H, Sykes B. Differential allelic expression of a fibrillin gene (FBN1) in patients with Marfan syndrome. Am $\mathcal{F}$ Hum Genet 1994;55:447-52.

27 Hewett DR, Lynch JR, Child A, Sykes BC. A new missense mutation of fibrillin in a patient with Marfan syndrome. $f$ Med Genet 1994:31:338-9.

28 Hayward C, Keston M, Brock DJH, Dietz HC. Fibrillin (FBN1) mutations in Marfan syndrome. Hum Mutat 1992;1:79. 
29 Hayward C, Rae AL, Porteous ME, Logie LJ, Brock DJ. Two novel EGF-like domains of the fibrillin gene (FBN1): SSCP screening of exons 15-21 in Marfan syndrome patients. Hum Mol Genet 1994;3:373-5.

30 Nijbroek G, Sood S, McIntosh I, et al. Fifteen novel FBN1 mutations causing Marfan syndrome detected by heteroduplex an 1995;57:8-21.

31 Wang $\mathrm{M}$, Wang $\mathrm{J}$, Imaizumi $\mathrm{K}$, et al. Three novel fibrillin mutations in exons 25 and 27: classical versus neonatal Marfan syndrome. Hum Mutat (in press)

32 Milewicz DM, Grossfield J, Cao S, et al. A mutation in FBN1 disrupts profibrillin processing and results in isolated skeletal features of the Marfan syndrome. 7 Clin Invest 1995;95:2373-8.

33 Lonnqvist L, Child A, Kainulainen $\mathrm{K}$, et al. A novel mutation of the fibrillin gene causing ectopia lentis. Genomics 1994;19:573-6.
34 Francke U, Berg MA, Tynan K, et al. A Gly1127Ser mutation in an EGF-like domain of the fibrillin-1 gene is a risk factor for ascending aortic aneurysm and dissection. $A m \mathcal{F}$ Hum Genet 1995;56:1287-96.

35 Tynan K, Comeau K, Pearson M, et al. Mutation screening of complete fibrillin-1 coding sequence: report of five new of complete fibrillin-1 coding sequence: report of five new Genet 1993;2:1813-21.

36 Karttunen L, Raghunath M, Lonnqvist L, Peltonen L. A compound-heterozygous Marfan patient: two defective fibrillin alleles result in a lethal phenotype. Am $\mathcal{F}$ Hum Genet 1994;55:1083-91.

37 Weyman AE. Complex congenital heart disease. I. A diagnostic approach. In: Principles and practice of echocardidiagnostic approach. In: Principles and practice of ech
ography. Philadelphia: Lea \& Febiger 1994:979-81.

38 Tsui LC. The cystic fibrosis transmembrane conductance regulator gene. Am $\mathcal{F}$ Respir Crit Care Med 1995;151 S47-53. 